\title{
1 Influence of smoking status on acute biomarker responses to 2 successive days of arduous military training
}

3 Andrew G.Siddall ${ }^{\mathrm{a}, \mathrm{b}}$, Keith A. Stokes ${ }^{\mathrm{a}}$, Dylan Thompson ${ }^{\mathrm{a}}$, Rachel M. Izard ${ }^{\mathrm{c}}$, Julie P.

4 Greeves $^{\mathrm{d}}$, James L. J. Bilzon ${ }^{\mathrm{a}}$.

5

$6 \quad$ aDepartment for Health, University of Bath, Bath, United Kingdom

7 bccupational Performance Research Group, University of Chichester, Chichester, United

8 Kingdom

9 'Department of Occupational Medicine, Army Recruiting and Initial Training Command, 10 Ministry of Defence, Upavon, United Kingdom

$11{ }^{\mathrm{d}}$ Army Personnel Research Capability, Ministry of Defence, Andover, United Kingdom

Corresponding author:

14 Professor James Bilzon, 1 West, Department for Health, University of Bath, Bath BA27NS

15 Email: j.bilzon@bath.ac.uk

16 Telephone: $+44(0) 1225383174$

Keywords Immune-inflammatory response; endocrine response; exercise; military; smoking, tobacco; acute

Funding: This work was funded by the Army Recruiting and Initial Training Command (formerly Army Recruiting and Training Division), UK Ministry of Defence: Army.

Declarations of Interests: None.

Acknowledgements: The authors would like to thank the staff at Infantry Training Centre, Catterick, for supporting this investigation and the trainees for their participation. 
27 Influence of smoking status on acute biomarker responses to successive days 28 of arduous military training

29

\section{Abstract}

Introduction: Habitual smoking is highly prevalent in military populations despite its association with poorer training outcomes. Smoking imposes challenges on immune and endocrine systems which could alter how smokers acutely respond to, and recover from, intensive exercise, particularly over multiple days of training.

Methods: Across a two-day period, thirty-five male British Army recruits (age $22 \pm 3 \mathrm{yr}$; mass $76.9 \pm 8.0$ $\mathrm{kg}$; height $1.78 \pm 0.06 \mathrm{~m} ; 15$ smokers) completed a $16.1 \mathrm{~km}$ loaded march (19.1 kg additional mass) on the first morning and a best-effort $3.2 \mathrm{~km}$ 'log race' (carrying a $60 \mathrm{~kg}$ log between six-to-eight people) on the subsequent morning. Blood samples were obtained upon waking and immediately post-exercise on both days and analysed for C-reactive protein (CRP), interleukin (IL)-6, testosterone/cortisol ratio and insulin-like growth factor (IGF)-1.

Results: Independent of smoking group, the exercise bouts on both days evoked significant increases in IL-6 $(\mathrm{p}<0.001)$ and decreases in testosterone/cortisol ratio $(\mathrm{p}<0.05)$. CRP concentrations on Day 2 were significantly higher than both time-points on Day $1(\mathrm{p}<0.001)$ and an $9 \%$ decline in IGF-1 occurred over the two-day period, but was not significant $(\mathrm{p}=0.063)$. No significant differences were observed between smokers and non-smokers ( $p>0.05)$.

Conclusions: Military-specific tasks elicited immune-inflammatory and endocrine responses, with systemic CRP and IGF-1 indicating that the physiological stress generated during the first training day was still evident on the second day. Despite the well-established impacts of smoking on resting levels of the markers examined, responses to two days of arduous military-specific training did not differ by smoking status. 


\section{Key messages}

- Smoking is prevalent in military populations and is linked to reduced physical fitness, heightened injury risk and poorer training outcomes.

- Habitual smoking imposes challenges on immune and endocrine systems, but whether this impacts on responses to exercise and recovery is unclear.

- Exercise evoked substantial inflammatory and hormonal responses during two days of military training, but neither resting nor exercise-induced levels were impacted by smoking status.

- The multi-stressor training environment, and the physical activity level and fitness of the population studied, may collectively explain the lack of smoking-related differences.

- Systemic CRP and IGF-1 indicated physiological stress from the first training day was evident in the second, suggesting continued training should implement sufficient recovery.

\section{Introduction}

The adverse impacts of tobacco smoking on health and risk of non-communicable diseases are widely recognised. Within military populations, habitual smoking is more prevalent than in the general public $^{1}$ and is associated with other adverse implications such as reduced physical fitness, heightened injury risk and poorer training outcomes ${ }^{2,3}$. These findings have brought into question what role longterm smoking plays in possible maladaptive responses to exercise and physical training ${ }^{4}$. Habitual smoking elicits a myriad of alterations in immune-inflammatory processes and hormonal control, which are implicated in the development of cardiovascular, metabolic and respiratory diseases ${ }^{5,6}$. Indeed, even in young, physically active adults, smokers tend to display elevated levels of oxidative stress and inflammation both at rest, which has been observed during long-term military training ${ }^{4}$, and in response to single bouts of laboratory-based exercise ${ }^{7-9}$. These responses indicate that habitual smoking has the potential to alter how smokers acutely respond to, and recover from, exercise but remains unsubstantiated.

Military training, and particularly short-term training exercises, are necessarily arduous to prepare personnel for their occupational role. It is customary for these operational simulations to span multiple days, where soldiers must repeatedly perform effectively while exposed to multiple stressors 
including sleep restriction, environmental extremes and/or energy deficit, which impose significant challenges on immune and endocrine systems ${ }^{10,11}$. Specifically, increases in pro-inflammatory cytokines such as interleukin (IL)-6 and elevated circulatory cortisol have been observed in a range of training durations $^{11,12}$, alongside a steady decline in basal insulin-like growth factor (IGF)-1 over a period of days ${ }^{13}$, akin to intensive periods of athletic overtraining. Though causally and mechanistically different, these alterations are comparatively similar to the immunosuppressive and inflammatory state observed chronically as a result of long-term smoking. Habitual smokers typically exhibit chronic low-grade inflammation, characterised by elevated levels of circulatory cytokines and acute-phase marker Creactive protein $(\mathrm{CRP})^{6,8,14}$, which play a key role in reduced secretion of IGF-1 $1^{15}$ and increased production of cortisol ${ }^{16}$ compared to non-smokers. For habitual smokers in the military, the combination of these underlying consequences of long-term smoking with exposure to external training stresses may present a cumulative physiological challenge.

Physical exercise transiently increases pro-inflammatory signalling ${ }^{17}$, stimulating an increase in CRP in the hours after exercise ${ }^{18}$, but is accompanied by anti-inflammatory actions which are implicated in the well-established long-term health benefits of regular physical activity ${ }^{17}$. Few studies have examined acute biochemical responses to exercise in habitual smokers compared to non-smokers, and have predominantly focused either on oxidative stress, which is mechanistically involved in the inflammatory profile of smokers ${ }^{14}$, or immune-inflammatory changes within exercise laboratory settings $^{7-9}$. Specifically, in response to low-moderate intensity exercise, young ( $\left.\sim 22 \mathrm{yr}\right)$ male smokers exhibited higher levels of inflammatory cytokines IL-1 receptor agonist and IL-6 than their age-matched non-smoking counterparts both immediately- and 1 hour-post-exercise ${ }^{8}$. In another young ( 24 yr) male cohort, an exacerbated oxidative stress response to graded cycling was observed in smokers ${ }^{7}$. To the authors' knowledge, acute hormonal responses to exercise in smokers and non-smokers have not been examined. During 10 weeks of British Army basic training, despite evidence of higher inflammation and oxidative stress in smokers, testosterone, cortisol and IGF-1 did not differ by smoking status ${ }^{4}$. As this study only examined waking (at-rest) samples however, it is still unknown whether more short- 
term, exercise-induced responses would differ in smokers in this population or whether these differences are evident further into recovery, such as in successive days of training.

Taken together, smoking causes noticeable alterations in biochemical markers and processes which also, in response to exercise, could be indicative of greater physiological $\operatorname{strain}^{13,17}$. Whether smokers respond differently to exercise, in a military-specific context, would be of particular interest given the combination of high smoking prevalence and intensive physical training that uniquely exists in the military. The aim of this study was therefore to examine acute inflammatory and hormonal responses to arduous bouts of military-specific exercise on two consecutive days and investigate whether these responses differed between habitual smokers and non-smokers. Based on the available evidence, our hypotheses were that, compared to non-smokers, smokers would present with higher inflammation at baseline and would have amplified exercise-induced immune-inflammatory responses and reduction in testosterone/cortisol ratio, in addition to greater training-induced decline in IGF-1 over the two-day period.

\section{Methods}

Data collection for this study was completed at the British Army's Infantry Training Centre, Catterick (ITC(C)), UK. Thirty-five British Army recruits (age $22 \pm 3$ yr; mass $76.9 \pm 8.0 \mathrm{~kg}$; height $1.78 \pm 0.06$ m) undertaking the Parachute regiment selection week at ITC(C) gave written informed consent to take part in the study. All participants were given a full written and verbal brief of the research study in the week prior to selection week. During the study, participants completed training according to their standard programme with only minor modifications, agreed with directing staff, to ensure data did not affect training. The study was approved by the Ministry of Defence Research Ethics Committee (MODREC/0911/236).

The parachute regiment selection week commences in week 19 of the regiment's 26-week training course at $\operatorname{ITC}(\mathrm{C})$ and is designed to assess recruit operational readiness by examining performance in a selection of arduous physical tasks which simulate operational stress and test various 
components of physical fitness. The study took place over the first two days of the selection week, containing a military exercise task on each morning: the '10-miler' on day 1 and the 'log race' on day 2. The 10-miler required recruits to complete a paced $16.1-\mathrm{km}$ march over varying terrain within 1 hour and 50 minutes $\left(8.8 \mathrm{~km} \cdot \mathrm{h}^{-1}\right)$ while carrying a 'Bergen' (backpack), webbing and rifle (total mass of 19.1 $\mathrm{kg}$ ). The $\log$ race required recruits in groups of 6-8 to carry a $60 \mathrm{~kg}$ log over approximately $3.2 \mathrm{~km}$ of varying terrain in as short a time as possible (and within 18 minutes; $\geq 10.7 \mathrm{~km} \cdot \mathrm{h}^{-1}$ ). Both events started at approximately $0900 \mathrm{hrs}$, after the participants had consumed breakfast and completed a standardised warm-up.

Anthropometric data and smoking status were assessed on the day prior to commencement of selection week. Body mass (weighing scales; Seca, Hamburg, Germany) and stature (stadiometer; Leicester, UK) were measured, and body fat percentage was estimated using measurements of skin-fold thickness ${ }^{19}$ on four upper-body sites (Biceps brachii, triceps brachii, sub-scapular and supra-iliac) using callipers (Holtain LTD. Crymych, UK). Smoking behaviour (history, frequency) and status were collected via a previously validated lifestyle questionnaire ${ }^{20}$ where habitual smokers were defined as those who regularly smoked $>1$ cigarette per day and non-smokers had never smoked.

Venous blood samples $(\sim 20 \mathrm{~mL})$ were drawn upon waking $(0500-0600 \mathrm{hrs})$ on both days after an overnight fast and immediately following both the 10-miler (Post-10) on day 1 and the log race (PostLR) on day 2. Blood samples were taken by venepuncture (antecubital vein) using a needle and Vacutainer system (BD Diagnostics, Becton, Dickinson \& Co.). Samples were collected in plain tubes (BD Diagnostics, Becton, Dickinson \& Co.) and left to clot for 60 minutes before being centrifuged to separate the serum. All samples were aliquoted and stored at $-80^{\circ} \mathrm{C}$ for analysis of blood chemistry. Commercially available enzyme immunoassays were used to determine serum concentrations of cortisol and IGF-1 (Diagnostic Systems Laboratories Inc., Webster, Texas, USA) and CRP and IL-6 (R\&D Systems Inc., Abingdon, UK). Combined intra- and inter-assay coefficient of variance calculated from study assay data for IL-6, CRP, cortisol, IGF-1 and TES were $2.22 \%, 2.00 \%, 1.02 \%, 2.56 \%$ and $1.35 \%$, respectively. 

measures design, assuming a medium effect of smoking or time $(\mathrm{f}=0.25)$, estimated a requirement for 18 participants per group to achieve sufficient power with statistical significance defined as $\mathrm{p} \leq 0.05$. Statistical analyses were performed using SPSS software (Version 22.0, IBM, USA). Independent ttests were performed on baseline anthropometric data to identify any initial between-group differences. A two-way mixed model analysis of variance (ANOVA), with effect sizes (partial eta-squared; $\eta_{p}^{2}$ ), was used to identify significant main effects of time, group or interaction in biochemical variables. As group sample numbers were uneven in this investigation, Greenhouse-Geisser output statistics were used. In the event of a significant interaction or training effect, post-hoc analysis with bonferroni adjustment was used to determine the location of the significant difference. Population characteristics are presented as mean \pm SD. Biochemical data are presented as mean $\pm 95 \%$ confidence intervals $(\mathrm{CI})$.

\section{Results}

Participant characteristics and anthropometric data organised by group are presented in Table 1. The non-smoking and smoking groups comprised 20 and 15 recruits, respectively. The smoking group had a mean $( \pm \mathrm{SD})$ cigarette consumption of $11.8( \pm 5.3)$ per day for an average of $7.0( \pm 2.8)$ years. No significant differences in anthropometric data were present between groups at baseline $(\mathrm{p}>0.05)$. between smokers and non-smokers $\left(\mathrm{CRP}: \mathrm{F}_{(1,33)}=0.11, \mathrm{p}=0.74, \eta_{p}^{2}=0.003\right.$; IL-6: $\mathrm{F}_{(1,33)}=0.08, \mathrm{p}=0.77$, $\left.\eta_{p}^{2}=0.002\right)$ and no interaction effects were identified in either marker $\left(\mathrm{CRP}: \mathrm{F}_{(1.03,34.06)}=0.01, \mathrm{p}=0.92\right.$ $\eta_{p}^{2}<0.001$; IL-6: $\left.\mathrm{F}_{(1.07,35.25)}=0.22, \mathrm{p}=0.66, \eta_{p}^{2}=0.006\right)$. Both markers, independent of smoking status, were significantly affected by training (main effect of time), but with different time-courses. CRP concentrations $\left(\mathrm{F}_{(1.03,34.06)}=45.51, \mathrm{p}<0.001, \eta_{p}^{2}=0.580\right)$ were significantly higher at both time points on the second day than both time points on the first $(\mathrm{p}<0.001)$. In contrast, IL-6 concentrations $\left(\mathrm{F}_{(1.07}\right.$, $\left.35.25)=80.98, \mathrm{p}<0.001, \eta_{p}^{2}=0.710\right)$ increased transiently in response to each exercise, where post-exercise values (Post-10 and Post-LR) were significantly higher than their respective pre-exercise values (Pre- 

after the 10-miler was 3.7 fold higher than after the $\log$ race $(\mathrm{p}<0.001)$. identified (Testosterone: $\mathrm{F}_{(2.48,81.81)}=1.78, \mathrm{p}=0.17, \eta_{p}^{2}=0.051$; Cortisol: $\mathrm{F}_{(1.61,52.96)}=0.207, \mathrm{p}=0.77$, $\eta_{p}^{2}=0.006$ ). Testosterone/cortisol ratio (Figure 1; Panel c) significantly reduced in response to both exercise bouts (main effect of time: $\mathrm{F}_{(1.81,59.65)}=14.47, \mathrm{p}<0.001, \eta_{p}^{2}=0.305$ ): a product of significant exercise-induced increases in cortisol (mean change $\pm 95 \% \mathrm{CI}$; Day 1: $+236 \pm 211 \mathrm{nmol} \cdot \mathrm{L}^{-1}, \mathrm{p}=0.004$; Day 2: $\left.+102 \pm 96 \mathrm{nmol} \cdot \mathrm{L}^{-1}, \mathrm{p}=0.005\right)$ and decreases in serum testosterone concentration (Day 1: -1.51 $\pm 0.55, \mathrm{p}<0.001$; Day 2: $\left.-1.82 \pm 0.57 \mathrm{ng} \cdot \mathrm{mL}^{-1} ; \mathrm{p}<0.001\right)$ on both days. 87.70) $=1.05, \mathrm{p}=0.37, \eta_{p}^{2}=0.031$ ) were present for serum IGF-1 (Figure 1; Panel d). Group average IGF-1 concentration steadily reduced from waking Pre-10 on day 1 to Post-LR on day 2 (mean change \pm 95\%CI: $\left.-16.5 \pm 9.0 \mathrm{ng} \cdot \mathrm{mL}^{-1}\right)$ but the main effect of time was not significant $\left(\mathrm{F}_{(2.66,87.70)}=2.61, \mathrm{p}=0.063\right.$ $\left.\eta_{p}^{2}=0.073\right)$

\section{Discussion}

The primary aim of this study was to assess, in a military population where smoking and exercise training are both common, whether habitual smokers appear to respond differently to arduous training. Inflammatory and endocrine markers were examined in smokers and non-smokers upon waking and after intense bouts of exercise during two days of military training. These markers are commonly altered by habitual smoking and, in response to a period of training, can reflect the magnitude of physiological strain experienced. The key finding of this study was that while biochemical markers reflected the arduous nature of training, responses did not differ by smoking status. Exercise on both days acutely increased IL-6 and cortisol, with subsequent decreases in testosterone/cortisol ratio. The arduous 
exercise on day 1 was reflected in elevated CRP concentrations upon waking on day 2 . An $8 \%$ decline in IGF-1 was observed over the two-day period which, in combination with the other observations, suggests a cumulative effect of the first day of training on the second. However, similar levels between groups in all markers, both at baseline and in response to training, suggest any influence of long-term smoking was not apparent.

Habitual smoking is highly prevalent in military training populations despite known impacts of smoking on health. To date, few studies have examined the acute responses of immune-inflammatory markers to exercise in smokers and non-smokers ${ }^{8,9}$, and no studies have investigated this research question with hormonal parameters, in a military population, or over successive days. The main finding of the current study was that smokers and non-smokers did not respond differently to either bout of exercise in any of the biochemical parameters measured. This is not consistent with previous studies that have demonstrated augmented cytokine and oxidative stress responses to, respectively, low-tomoderate- and incremental intensity exercise in smokers ${ }^{7,8}$. The sparsity of current literature however, means it is unclear whether higher intensity exercise (such as that performed in the current study) would elicit a magnitude of response that would mask any differences between smokers and non-smokers, rather than highlight them. The current study hypothesis was also, in part, based on the common finding that chronic smokers exhibit an elevated inflammatory profile at rest, which could theoretically act to prime and/or exacerbate the immune-inflammatory response to exercise ${ }^{8,9}$. Higher resting oxidative stress and CRP have been observed in smokers during initial military training, in a British Army recruit cohort comparable to the present study ${ }^{4}$. Given this evidence, and that systemic inflammation is exacerbated by oxidative stress ${ }^{14}$, similar resting inflammation observed between groups was surprising, but may have contributed to the similar immune-exercise response. The well-recognised antiinflammatory effect of long-term habitual exercise may also have contributed ${ }^{17}$, since the participating recruits were 19 weeks into a training course, potentially counteracting low-grade inflammation normally observed in untrained smokers ${ }^{14}$. Prior to this investigation, it was difficult to ascertain whether hormone responses to exercise would differ by smoking status and in which relative direction due to lack of available evidence. Numerous mechanisms linked to the actions of nicotine and immune- 
inflammatory signalling have been implicated in altered resting hormone levels in smokers previously ${ }^{16}$. However, the current study did not provide further evidence of this nor indicate a discernible impact on training-induced endocrine responses.

Military field exercise, involving consecutive days of arduous training, has been shown to elicit alterations in hormone concentrations similar to the present study, but typically over longer durations and in energy deficit. Specifically, suppression of IGF-1 and testosterone alongside increased circulating concentrations of cortisol have been demonstrated during periods of intensive military training ${ }^{12,21}$. Increases in cortisol, in particular, are associated with daily and weekly training volume $\mathrm{e}^{10,21}$ and sleep disruption ${ }^{22}$. Taken together, the findings of previous research, suggest that military field exercise evokes a period of metabolic stress that would be, ostensibly, maladaptive if prolonged, and that endocrine responses can act as indicators of the strain experienced ${ }^{13,21,23}$. The exercise-induced elevations in cortisol and $~ 9 \%$ decline in IGF-1 we observed over the two-day period, also support this notion. While the study setting was not a field exercise, the exercise tasks are designed to simulate military-specific operational stress. Unfortunately, energy balance and/or sleep patterns were not examined and it is therefore not possible to discern whether the observed decline in IGF-1 was evoked solely by physical demand of the exercise itself despite sufficient recovery and caloric intake ${ }^{23}$, or as a combination of arduous training, insufficient recovery and/or energy deficit ${ }^{24}$. While the current study is short in duration, the patterns identified reflect that an extension of training of this nature warrant suitable recovery strategies to avoid overtraining 22 .

Our observations are consistent with previous evidence that IL-6 transiently increases in response to exercise and that the magnitude of this response is affected by exercise intensity and duration ${ }^{17}$. The increase in IL-6 concentrations in response to the 10-miler (1 hour 50-minute duration) was almost four-fold larger than to the log race $(<20$-minute duration). The relative difference between these responses could simply be a function of time since onset of exercise, or could indicate that duration of exercise had a greater effect on the inflammatory response than exercise intensity. Also in agreement with previous literature, CRP concentrations did not change immediately in response to exercise, but were significantly elevated by the second morning. This is consistent with the typical rise in CRP 
associated with exercise (stimulated by IL-6) which can continue to increase over 24 hours $^{18}$. In the current study, the log race was initiated when waking CRP concentration averaged greater than $3 \mathrm{mg} \cdot \mathrm{L}^{-}$

${ }^{1}$; higher than would be expected in a normal healthy population of this age and cardiorespiratory fitness. From the magnitude and nature of the responses observed, it is possible that the multi-stressor environment of military training means the impacts of smoking were too small to be independently identified, particularly with the sample size available.

Due to the high prevalence of smoking in military populations, it seemed appropriate to examine this research question with an ecologically valid design, by observing successive days of military-specific training with no modification to the training programme. However, this did also result in key limitations of the current study. This study was a part of a larger programme of work with different research aims that required waking samples, meaning (immediately) pre-exercise samples or a higher frequency of blood sampling could not be completed without substantial disruption to training. This, and examining further subsequent days of training, could have helped explain some of the observed responses. For instance, it is plausible that our waking samples reflect early morning peak/nadir concentrations of markers such as testosterone, against which post-exercise concentrations appear substantially reduced. Similarly, Kastelein et al. (2015) observed elevated cytokines in smokers during 1-4 hours of post-exercise recovery, which may have occurred in the current study but was not observable due to the study design.

\section{Conclusions}

We observed that consecutive days of arduous physical exercise evoked marked inflammatory and endocrine responses, but that these responses were not influenced by smoking status. Previous laboratory studies have demonstrated differing responses of oxidative stress and immune-inflammatory markers to exercise between smokers and non-smokers ${ }^{7-9}$, and it is possible that with the greater experimental control afforded by a laboratory setting, some differences may have been apparent. However, a high incidence of smoking during long-term exercise training is unique to the military and the study aimed to assess an ecologically valid representation of military-specific exercise. It is unclear 

future investigations could look to understand whether being highly physically fit and/or active is beneficial to immune health amongst smoking populations.

\section{References}

1. Robinson M, Siddall A, Bilzon J, et al. Low fitness, low body mass and prior injury predict injury risk during military recruit training: a prospective cohort study in the British Army. BMJ Open Sport Exerc Med 2016;2:e000100.

2. Siddall AG, Bilzon JLJ, Thompson D, et al. Smoking status and physical fitness during initial military training. Occup Med (Lond) 2017.

3. Brooks RD, Grier T, Dada EO, et al. The combined effect of cigarette smoking and fitness on injury risk in men and women. Nicotine Tob Res 2018.

4. Siddall A, Bilzon J, Thompson D, et al. Smoking and Biochemical, Performance, and Muscle Adaptation to Military Training. Medicine \& Science in Sports \& Exercise 2020;52:1201-1209.

5. Taylor JD. COPD and the response of the lung to tobacco smoke exposure. Pulm Pharmacol Ther

6. Sopori M. Effects of cigarette smoke on the immune system. Nat Rev Immunol 2002;2:372-377.

7. Bloomer RJ, Creasy AK, Smith WA. Physical work-induced oxidative stress is exacerbated in young cigarette smokers. Nicotine Tob Res 2007;9:205-211.

8. Kastelein TE, Duffield R, Marino FE. Acute Immune-Inflammatory Responses to a Single Bout of Aerobic Exercise in Smokers; The Effect of Smoking History and Status. Front Immunol 2015;6. Available at: http://www.ncbi.nlm.nih.gov/pmc/articles/PMC4688366/. Accessed November 1, 2016.

9. Kastelein TE, Donges CE, Mendham AE, et al. The Acute Exercise-Induced Inflammatory Response: A Comparison of Young-Adult Smokers and Nonsmokers. Research Quarterly for Exercise and Sport 2017;88:15-25.

10. Tanskanen MM, Kyröläinen H, Uusitalo AL, et al. Serum sex hormone-binding globulin and cortisol concentrations are associated with overreaching during strenuous military training. J Strength Cond Res 2011;25:787-797.

11. Diment BC, Fortes MB, Greeves JP, et al. Effect of daily mixed nutritional supplementation on immune indices in soldiers undertaking an 8-week arduous training programme. Eur J Appl Physiol 2012;112:14111418.

12. Kyröläinen H, Karinkanta J, Santtila M, et al. Hormonal responses during a prolonged military field exercise with variable exercise intensity. Eur J Appl Physiol 2008;102:539-546.

13. Nindl BC, Pierce JR. Insulin-like growth factor I as a biomarker of health, fitness, and training status. Med Sci Sports Exerc 2010;42:39-49.

14. Helmersson J, Larsson A, Vessby B, et al. Active smoking and a history of smoking are associated with enhanced prostaglandin F(2alpha), interleukin-6 and F2-isoprostane formation in elderly men. Atherosclerosis 2005;181:201-207. 
15. Renehan AG, Atkin WS, O'dwyer ST, et al. The effect of cigarette smoking use and cessation on serum insulin-like growth factors. Br J Cancer 2004;91:1525-1531.

16. Steptoe A, Ussher M. Smoking, cortisol and nicotine. Int J Psychophysiol 2006;59:228-235.

17. Petersen AMW, Pedersen BK. The anti-inflammatory effect of exercise. J Appl Physiol 2005;98:1154-1162.

18. Plaisance EP, Grandjean PW. Physical activity and high-sensitivity C-reactive protein. Sports Med 2006;36:443-458.

19. Durnin JV, Womersley J. Body fat assessed from total body density and its estimation from skinfold thickness: measurements on 481 men and women aged from 16 to 72 years. Br J Nutr 1974;32:77-97.

20. Robinson M, Stokes K, Bilzon J, et al. Test-retest reliability of the Military Pre-training Questionnaire. Occup Med (Lond) 2010;60:476-483.

21. Tyyskä J, Kokko J, Salonen M, et al. Association with physical fitness, serum hormones and sleep during a 15-day military field training. J Sci Med Sport 2010;13:356-359.

22. Booth CK, Probert B, Forbes-Ewan C, et al. Australian army recruits in training display symptoms of overtraining. Mil Med 2006;171:1059-1064.

23. Rarick KR, Pikosky MA, Grediagin A, et al. Energy flux, more so than energy balance, protein intake, or fitness level, influences insulin-like growth factor-I system responses during 7 days of increased physical activity. J Appl Physiol 2007;103:1613-1621. 
359 Table 1. Participant characteristics by group. Values are means \pm SD.

\begin{tabular}{|c|c|c|c|}
\hline \multirow[b]{2}{*}{ Variable } & \multicolumn{3}{|c|}{ Smoking Status } \\
\hline & Non-smokers $(\mathrm{n}=20)$ & Smokers $(n=15)$ & All $(n=35)$ \\
\hline Age (yr) & $22 \pm 3$ & $22 \pm 3$ & $22 \pm 3$ \\
\hline Body mass (kg) & $77.8 \pm 8.9$ & $75.9 \pm 6.9$ & $76.9 \pm 8.0$ \\
\hline Height (m) & $1.78 \pm 0.07$ & $1.77 \pm 0.05$ & $1.78 \pm 0.06$ \\
\hline Body Fat (\%) & $14.2 \pm 2.7(n=18)$ & $12.7 \pm 2.1(n=13)$ & $13.6 \pm 2.6$ \\
\hline
\end{tabular}

360

361

362

363

364

365

366

367

368

369

370

371

372

373

374

375 

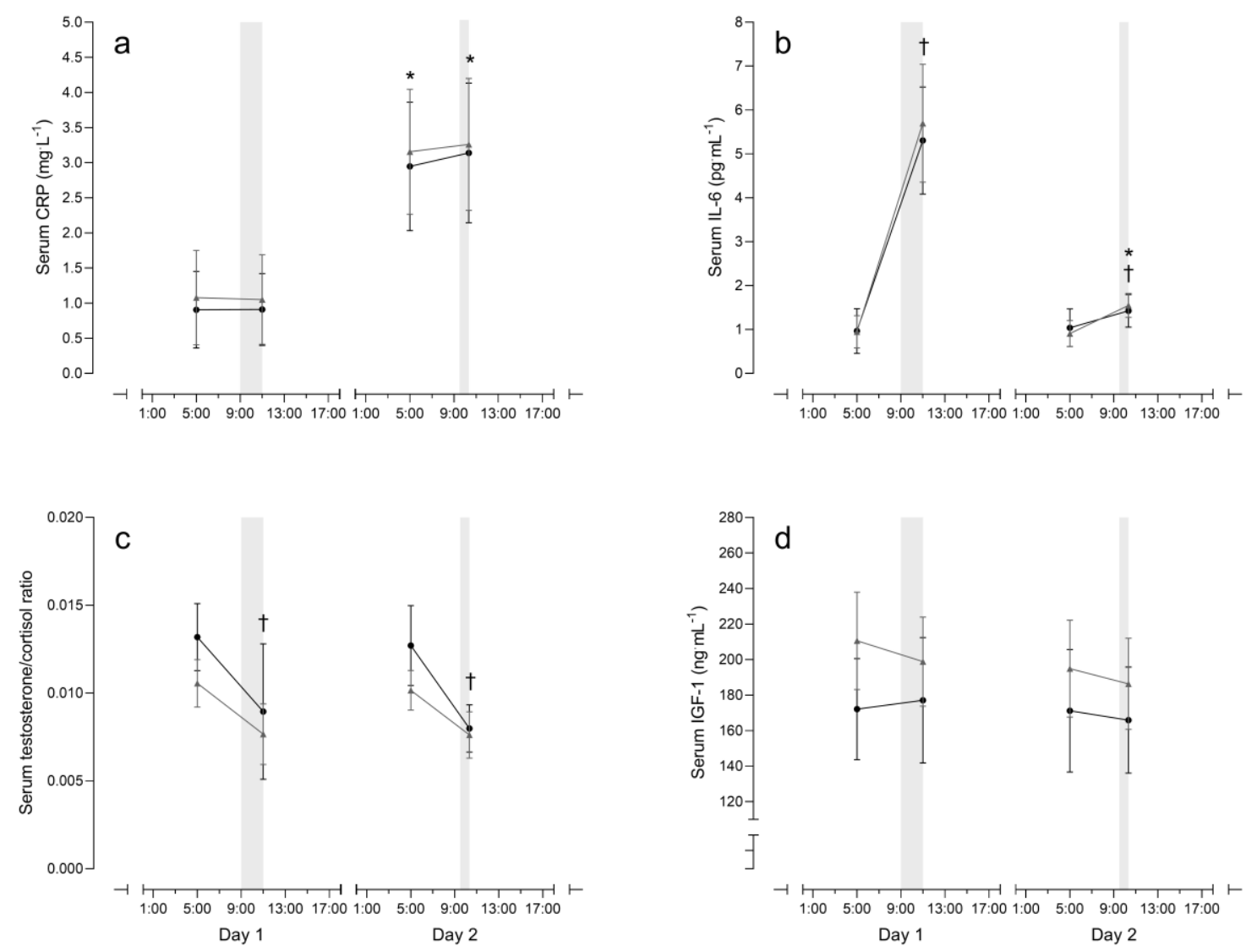

377 Fig 1. Effects of two days of simulated operational stress on serum concentrations of inflammatory and hormonal

378 markers. Mean $( \pm 95 \% \mathrm{CI})$ serum concentration of CRP $\left(\mathrm{mg} \cdot \mathrm{L}^{-1} ; \mathbf{a}\right), \mathrm{IL}-6\left(\mathrm{pg} \cdot \mathrm{mL}^{-1} ; \mathbf{b}\right)$, testosterone to cortisol ratio $(\mathbf{c})$ and 379 IGF-1 (ng. $\left.\mathrm{mL}^{-1} ; \mathbf{d}\right)$ between non-smokers (Grey triangle) and smokers (Black circle). Grey boxes denote exercise: '10-miler' 380 on Day 1 and 'Log Race' on Day 2. *Different from equivalent time point on Day $1(\mathrm{p}<0.05)$. $\dagger$ Different from pre-exercise $(\mathrm{p}<0.05)$ 